\title{
High-latitude HF Doppler observations of ULF waves. 1. Waves with large spatial scale sizes
}

\author{
D. M. Wright, T. K. Yeoman, P. J. Chapman \\ Department of Physics and Astronomy, University of Leicester, University Road, Leicester, LE1 7RH, UK
}

Received: 10 February 1997 / Revised: 30 May 1997 / Accepted: 9 June 1997

\begin{abstract}
A quantitative study of observations of the ionospheric signatures of magnetospheric ultra low frequency (ULF) waves by a high-latitude (geographic: $69.6^{\circ} \mathrm{N} 19.2^{\circ} \mathrm{E}$ ) high-frequency Doppler sounder has been undertaken. The signatures, which are clearly correlated with pulsations in ground magnetometer data, exhibit periods in the range $100-400 \mathrm{~s}$ and have azimuthal wave numbers in the range 3-8. They are interpreted here as local field line resonances. Phase information provided by $\mathrm{O}$ - and X-mode Doppler data support the view that these are associated with field line resonances having large azimuthal scale sizes. The relative phases and amplitudes of the signatures in the Doppler and ground magnetometer data are compared with a model for the generation of Doppler signatures from incident ULF waves. The outcome suggests that the dominant mechanism involved in producing the Doppler signature is the vertical component of an $\boldsymbol{E} \times \boldsymbol{B}$ bulk motion of the local plasma caused by the electric field perturbation of the ULF wave.
\end{abstract}

Key words Auroral ionosphere $\cdot$ Magnetosphereionosphere interactions - MHD waves and instabilities HF Doppler · ULF Waves

\section{Introduction}

Ultra low frequency (ULF) waves are an important coupling mechanism between the magnetosphere and the ionosphere, since they transfer both energy and momentum. These processes are most significant in the high-latitude ionosphere, where the magnetosphereionosphere interaction is strongest. The waves also act

Correspondence to: D. M. Wright

e-mail: dmw7@ion.le.ac.uk as an important diagnostic of magnetospheric morphology and dynamics. The ionosphere determines the boundary conditions (e.g. Yeoman et al., 1990) for magnetospheric magnetohydrodynamic (MHD) wave modes and hence controls the transfer of energy and momentum. It also modifies the magnetospheric wave signature, leading to rotation and attenuation of the wave magnetic signature detected on the ground (e.g. Hughes and Southwood, 1976; Hughes, 1983). The ionospheric signature of ULF waves is thus an important topic of study.

Up to the present the principal instruments for the investigation of the ionospheric signatures of ULF waves have been coherent-scatter radars. There are many reports of the ionospheric signatures of field line resonances in both the E-region, using VHF radar data (e.g. Walker et al., 1979; Yeoman et al., 1990) and in the F-region, using high-frequency (HF) radar data (e.g. Ruohoniemi et al., 1991). They generally exhibit small effective azimuthal wave numbers $(m)$ typically in the range $0-20$, which is equivalent to azimuthal wavelengths of greater than $685 \mathrm{~km}$ at the latitude of Tromsø $\left(69.6^{\circ} \mathrm{N}\right)$. Non-resonant ULF wave features have also been observed by VHF radars (e.g. Allan et al., 1982, 1983). Fenwick and villard (1960) first suggested that geomagnetic variations might be associated with observed shifts in the frequency of ionospherically reflected radio signals collected from an HF Doppler sounder. Since then, short-period oscillations in the frequency of HF signals have been correlated with magnetospheric ULF pulsations measured by ground magnetometers (e.g. Jacobs and Watanabe, 1966; Klostermeyer and Röttger, 1976; Tedd et al., 1989; Menk, 1992). In recent years, an attempt has been made to model the mechanisms involved in generating the ionospheric signatures observed by HF Doppler sounders (Poole et al., 1988; Sutcliffe and Poole, 1989, 1990; Sutcliffe, 1994).

HF Doppler sounders offer an important experimental technique for investigating the ionospheric signatures of magnetospheric ULF waves. This type of radio diagnostic offers the ability to select the height at which 
the ionosphere is probed by changing the sounding frequency, as well as providing measurements of the ionosphere with high spatial and temporal resolution. A new sounder known as the Doppler Pulsation Experiment (DOPE), has recently been constructed in Leicester, UK and was deployed in May 1995 near Tromsø, Norway (geographic: $69.6^{\circ} \mathrm{N} 19.2^{\circ} \mathrm{E}$; geomagnetic: $\left.67.0^{\circ} \mathrm{N} 117.0^{\circ} \mathrm{E} ; L=6.3\right)$. It is located close to the European Incoherent Scatter (EISCAT) radar facility (e.g. Rishbeth and Williams, 1985) which enables comparison with EISCAT data from suitable common and special programs with the aim of investigating the ionospheric boundary conditions for MHD wave modes in the magnetosphere, studying the height profile of ULF wave signatures and for comparing observations with existing theoretical models and measurements made with other ground-based instruments. A limited number of observations of the ionospheric signatures of ULF waves with the EISCAT radar have been reported by Crowley et al. $(1985,1987)$ and Glangeaud et al. (1985).

This paper presents the first observations made by DOPE. The simultaneous detection of Pc4-5 pulsations at the ground and their signatures in the ionosphere at high latitudes have allowed the vertical and horizontal structure of the ULF waves to be investigated. Several events with large azimuthal scale sizes ( $m$ numbers in the range 3-8) have been studied quantitatively. They have been interpreted as localised field line resonances, and the observed Doppler signatures have been compared to a model for the generation of such signatures. It has been found that the vertical component of an $\boldsymbol{E} \times \boldsymbol{B}$ motion in the ionosphere, caused by the incident ULF wave, is the dominant mechanism involved.

\section{Instrumentation}

\subsection{The high-latitude Doppler sounder}

The DOPE sounder consists of a frequency stable transmitter (Chapman, 1995, 1997b) and a receiver (Chapman, 1997a) which have a ground-separation of about $50 \mathrm{~km}$, giving a near-vertical radio path roughly along a magnetic meridian. A fixed-frequency $(4.45$ $\mathrm{MHz}$ ) continuous wave signal is radiated and, after reflection in the F-region ionosphere, received at the ground. The Doppler technique utilises the fact that variations in the refractive index or bulk motion of the plasma along the path of the radio wave cause small shifts in the received frequency, due to changes in the phase path of the wave. Thus, the frequency shift, $\Delta f$, is given by

$$
\Delta f=-\frac{1}{\lambda} \frac{\mathrm{d} P}{\mathrm{~d} t},
$$

where $P$ is the phase path of the signal and $\lambda$ is the wavelength. The measured Doppler shifts, which are typically less than $1 \mathrm{~Hz}$, can thus indicate the characteristics of waves affecting the ionospheric plasma in the region being sounded. In the case where refractive index does not vary with time, this can be interpreted as an equivalent vertical motion of the reflection point with a velocity, $v$, using, for a vertical incidence sounder, the relation

$$
\Delta f=-2 \frac{v}{c} f,
$$

where $c$ is the speed of light and $f$ is the sounding frequency.

The spatial resolution of a sounder such as this is determined by the area over which it integrates data. This is given, to a first approximation, by the region of specular reflection of the HF radio wave from the ionosphere which is equal to the area of the first Fresnel zone of the reflecting plane of the ionosphere. A simplified relation for the radius of the first Fresnel zone, assuming an idealised mirror like reflection is given by (Georges, 1967)

$$
R=\sqrt{r_{0} \lambda},
$$

where $r_{0}$ is, for a vertical incidence sounder, the height of reflection and $\lambda$ is the free space wavelength of the diagnostic signal. For an F-region reflection height of $r_{0} \approx 250 \mathrm{~km}$ and a sounder frequency of $4.45 \mathrm{MHz}$ $(\lambda=67 \mathrm{~m}), R \approx 4 \mathrm{~km}$. When compared to the scale size of the integration area of ground magnetometers $(\geq 120 \mathrm{~km}$; Hughes and Southwood, 1976) and VHF coherent-scatter radars $(15-45 \mathrm{~km})$, it is clear that a Doppler sounder offers ionospheric observations with high spatial resolution. The EISCAT incoherent-scatter radar, however, has a beam width of the order of $2 \mathrm{~km}$ at F-region heights.

\subsection{Sensitivity as a function of sounder frequency}

The sensitivity of the HF Doppler technique to electron density changes increases as the sounder frequency approaches the ionospheric critical frequency (foF2). This is because small changes in the ionospheric electron density at the F-region peak lead to large changes in the group and phase path of the radio wave as it approaches its reflection point, and hence give a large Doppler frequency shift as indicated by Eq. (1) (e.g. Davies, 1990). The amplitude of the Doppler signature of an ULF wave would therefore appear larger when the HF reflection point is nearer the F-region peak. It has been suggested (Wright, 1996) that this is likely to have a significant effect on the number of observations of ULF wave signatures made by a HF Doppler sounder throughout the day.

\subsection{Data acquisition and analysis}

The Doppler sounder receiver system consists of a twin channel receiver and a PC employed to control receiver operations and to log data on a digital tape (Wright, 1996). The signal is received on a pair of crossed active dipole antennas and an $\mathrm{O}-\mathrm{X}$ mode discriminator enables $\mathrm{O}-$ and X-mode signals to be resolved on channels 1 and 2 of the receiver, respectively (Chapman, 1997a). The 
receiver mixes the signal input frequency down to a baseband level which represents the offset from the diagnostic frequency of $4.45 \mathrm{MHz}$. The receiver output is filtered with a cut off of $15 \mathrm{~Hz}$ at the $3 \mathrm{~dB}$ level. As far as the authors are aware, this is the first time continuous $\mathrm{O}$ - and X-mode observations of ULF wave signatures have been made at high latitudes. Previously, the $6 \mathrm{~h}$ of digital ionosonde observations of $\mathrm{Pc} 3-4$ pulsations recorded by Jarvis and Gough (1988) at the geomagnetically mid-latitude station of Halley, Antarctica employed O-X mode discrimination, as did the lowlatitude (Grahamstown, South Africa) observations of the ionospheric signatures of Pc3 pulsations reported by Sutcliffe and Poole (1984).

The data, which are sampled at the receiver output at a rate of $40 \mathrm{~Hz}$, undergo spectral analysis employing a fast Fourier transform (FFT) routine in order to produce a "Doppler trace" such as those reproduced in the top two panels of Fig. 1. The temporal resolution of these data is $12.8 \mathrm{~s}$, which is a result of consecutive blocks of 512 samples being Fourier analysed. Spectral components with values above some threshold (usually $50 \%$ of the peak value) are included on the Doppler trace and these represent the diagnostic signal frequency shift.

\subsection{Ground magnetometers}

In addition to the Doppler data presented in this paper, data from two ground magnetometer stations in northern Scandinavia are included. These stations are Tromsø (TRO), Norway and Sodankylä (SOD; geographic: $67.4^{\circ} \mathrm{N}, 26.6^{\circ} \mathrm{E}$ ), Finland which are both part of the IMAGE magnetometer chain (e.g. Lühr, 1994). These data have a time resolution of $10 \mathrm{~s}$. The resolution of magnetic field perturbation magnitude measured by
TRO is $1 \mathrm{nT}$, and therefore for a ground magnetic signature to be measured in association with ionospheric Doppler data a ground amplitude of at least $4 \mathrm{nT}$ peakto-peak is required. The resolution of the SOD data employed in this study is considerably better at $0.01 \mathrm{nT}$. Spatially separated stations offer the possibility to determine the latitudinal phase change of a ULF wave as well as its azimuthal phase change, characterised by the effective azimuthal wave number, $m$, the number of degrees of change in phase per degree of longitude on the Earth's surface. Due to the way in which ground magnetometers integrate information over an area with a scale length of the order of the E-region height (Hughes and Southwood, 1976), phase mixing of signals from adjacent regions in the ionosphere results in the attenuation of waves observed on the ground with high $m$-values (corresponding to small azimuthal scale lengths).

\section{Results}

This section presents Doppler observations of ULF waves for which an accompanying magnetic signature was detected on the ground. However, the DOPE sounder has also identified a population of ionospheric signatures of pulsations uncorrelated with ground magnetic observations. This population has been interpreted as being ULF waves with small azimuthal scale sizes, and will be reported in a subsequent paper.

Fig. 1 illustrates a typical pulsation event which occurred on 13 November 1995 and clearly displays an HF Doppler wave signature which has a correlated ground magnetic signature. $\mathrm{O}$ - and $\mathrm{X}$-mode Doppler data are presented as time-series, along with unfiltered $\mathrm{X}$-(north-south) and Y-(east-west) component magnetometer data for comparison. It is evident that three

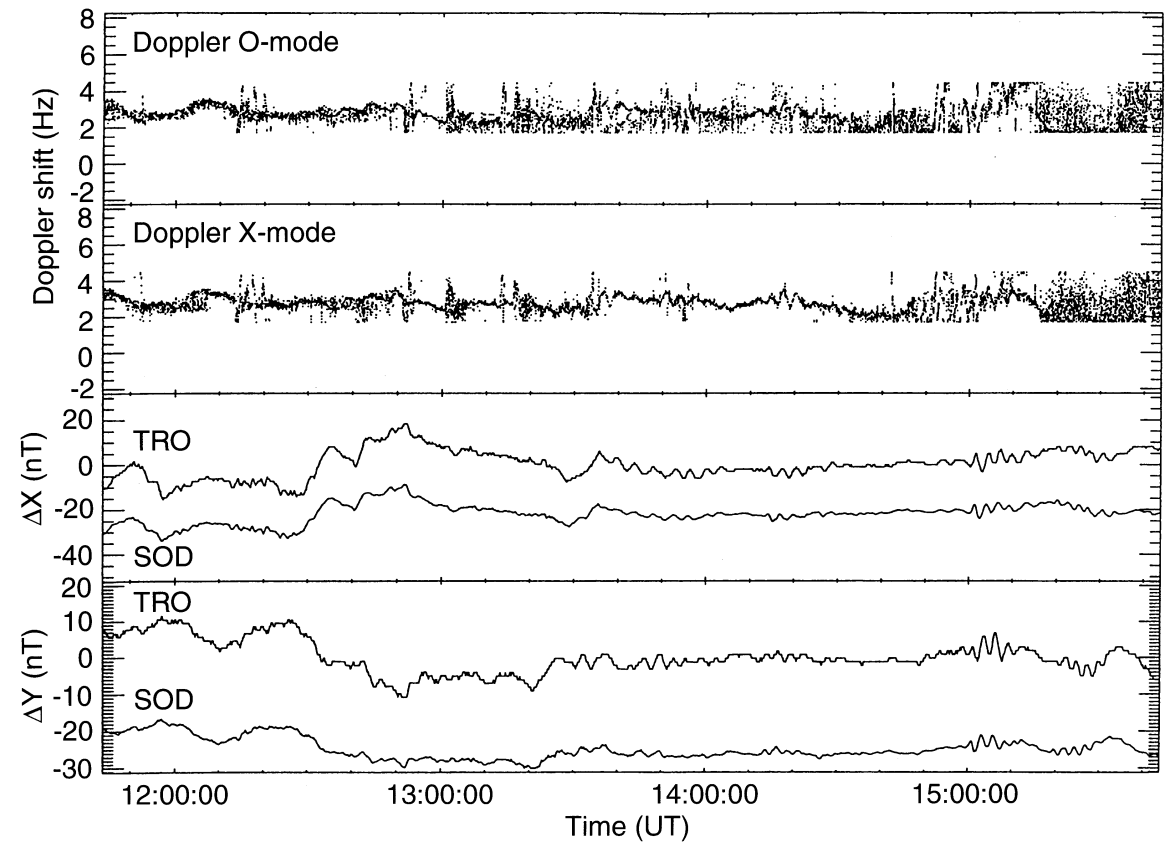

Fig. 1. Doppler and magnetometer data for the ULF wave event on 13 November 1995. The panels display (from the top down): Doppler O-mode frequency shift, X-mode frequency shift, TRO (upper trace) and SOD (lower trace) X component and $\mathrm{Y}$ component. The sampling interval of the Doppler data is $12.8 \mathrm{~s}$, and $10.0 \mathrm{~s}$ for that from the magnetometers. The Doppler data are plotted above a threshold of $50 \%$ of the amplitude of the main spectral component for each FFT. 


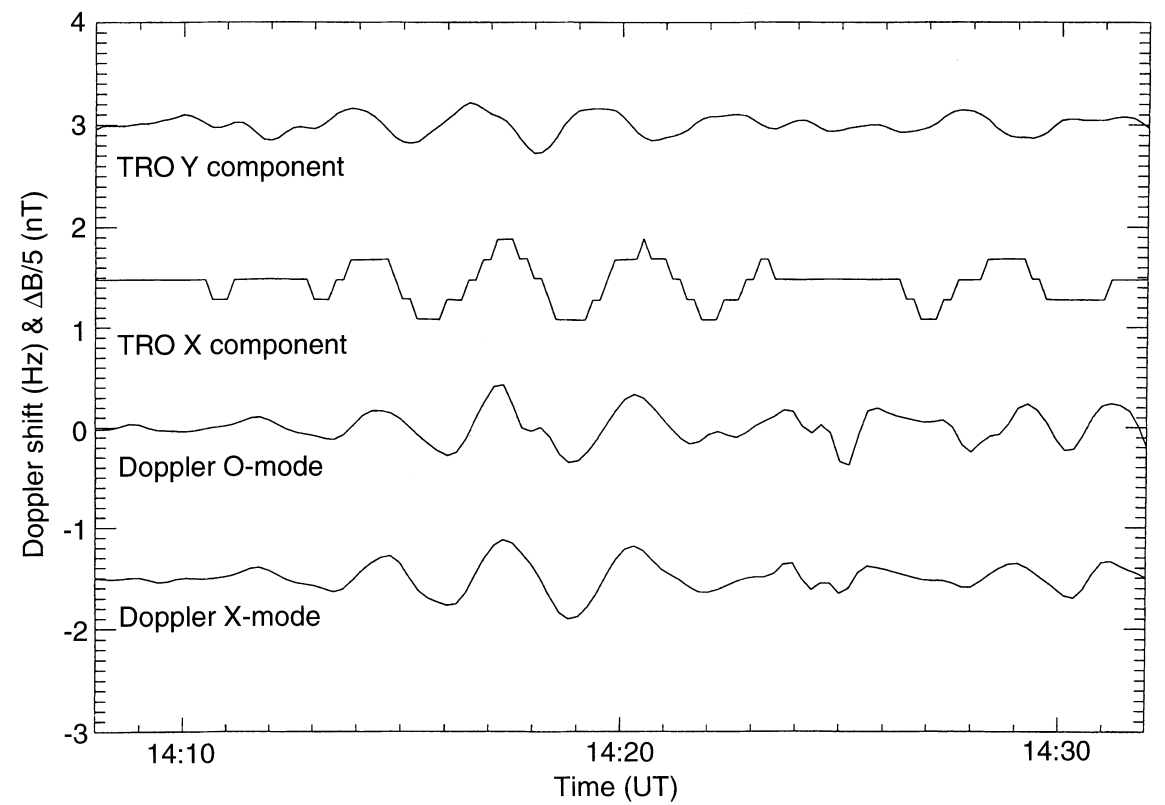

Fig. 2. Time-series of TRO magnetometer $X$ and $\mathrm{Y}$ components and peak traces for the $\mathrm{O}$ and X-mode signatures for the interval 14:0814:32 UT on 13 November 1995. Data are filtered to exclude periods outside the range 60 $300 \mathrm{~s}$. Note that the magnetometer component amplitude has been divided by 5 and that the ordinate axis is a relative scale and arbitrary offsets applied to each trace wave packets exist in the data. The packets commence at about 13:33, 14:10 and 15:00 UT, respectively. The Omode trace is considerably noisier than that of the $\mathrm{X}$ mode. During this event there was an interfering signal with a small frequency offset from our own signal. It had a mean level somewhere below $2 \mathrm{~Hz}$ on this plot. In order to remove the interferer the part of the spectrum in the range $1.5-4.5 \mathrm{~Hz}$ only has been plotted. In chronological order, the three packets contain 9, 5 and 4 discernible wave cycles in the Doppler data. Also presented on this plot are the unfiltered $\mathrm{X}$ - and $\mathrm{Y}$ components of the Tromsø (TRO) and Sodankylä (SOD) IMAGE magnetometer stations. They have a separation in longitude large enough to enable the calculation of the azimuthal wave number, $m$, of the ULF pulsations studied here. There is a clear wave signature in the magnetometer data at the same time as those in the Doppler data. The amplitude of the magnetometer signature is higher at TRO than at SOD. The Doppler oscillation has an amplitude of around $1 \mathrm{~Hz}$ peak-to-peak, which is equivalent to an oscillation of the reflection level with a peak velocity, determined from Eq. (2), of $34 \mathrm{~m} \mathrm{~s}^{-1}$ at this sounding frequency, and there is a corresponding fluctuation in the magnetometer data with an amplitude of around 5 nT peak-to-peak. Figure 2 shows a section of this data which has been filtered to include only those periods between 60 and $300 \mathrm{~s}$. The interval 14:10-14:30 UT was examined because it had the clearest $\mathrm{O}$-mode signature. The Doppler data in this figure is the peak trace of the spectra plotted in Fig. 1. This is necessary in order to reject noise and to create a single-component time-series on which a frequency analysis is possible. The TRO Ycomponent phase leads all the other signatures, and there is a clear correlation between all four time-series.

The filtered data in the interval 14:10-14:30 UT (Fig. 2) were passed through an FFT and the resulting power spectra, which have a frequency resolution of 0.83
$\mathrm{mHz}$, are given in Fig. 3. A clear sharp peak exists in both Doppler and magnetometer power spectra at a frequency of $5.8 \mathrm{mHz}$, which corresponds to a wave period of $172 \mathrm{~s}$,
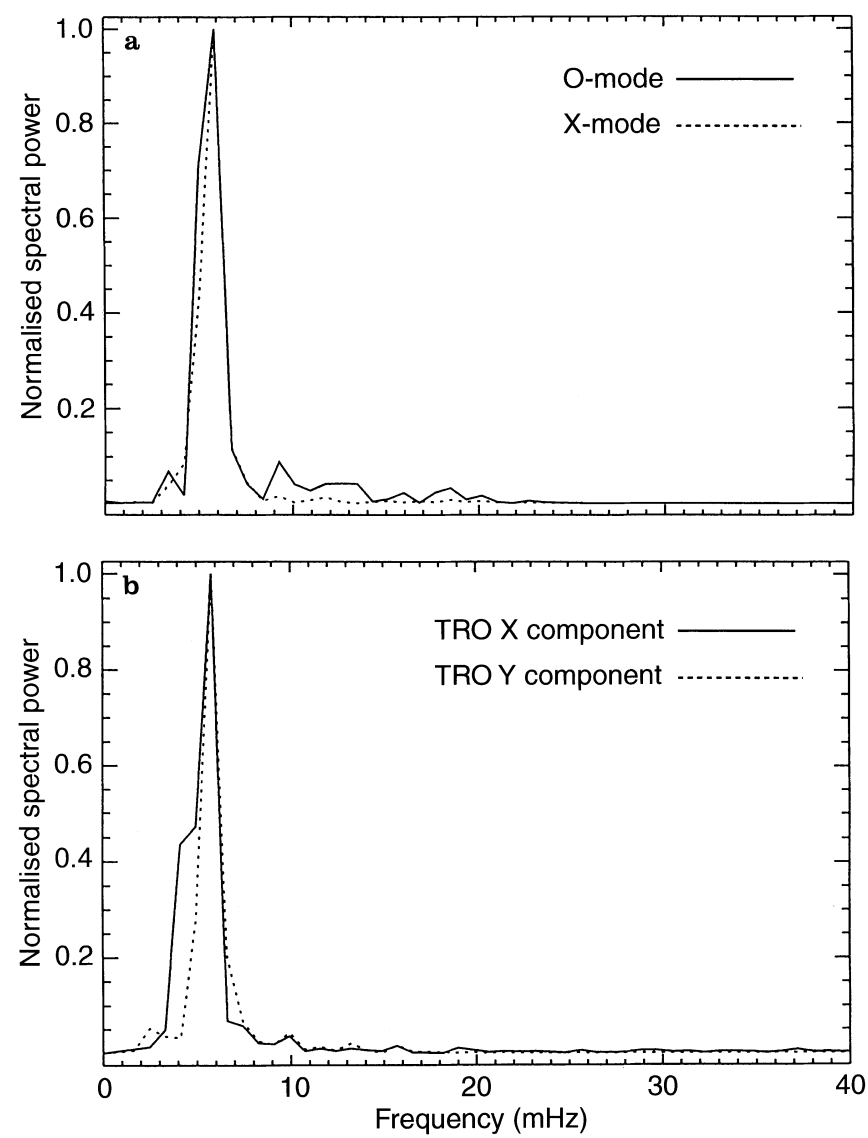

Fig. 3 a,b. Fourier power spectra of the time-series of a Doppler Oand $\mathrm{X}$-mode traces and $\mathbf{b}$ Tromsø magnetometer (TRO) $\mathrm{X}$ and $\mathrm{Y}$ components for the interval 14:10-14:30 UT on 13 November 1995 as illustrated in Fig. 2 
in the Pc5 frequency range (Jacobs et al., 1964). Comparison of the Fourier phase of the Y-component data of SOD and TRO reveals an azimuthal wave number of $6.4 \pm 2.7$ (equivalent to an azimuthal wavelength of about $2100 \mathrm{~km}$ ). The Y-component is selected to calculate $m$ because the phase of the wave in the X-component of two latitudinally separated magnetometers may vary considerably over a field line resonance (e.g. Orr, 1984), and thus the $\mathrm{Y}$-component is more phase stable in latitude. This is particularly important as TRO and SOD are latitudinally separated. A reasonable estimate of the error in the calculated value of $m$ is derived from the time resolution of the magnetometer data. For this interval the Fourier phase of the dominant spectral component for the X-mode signature leads the O-mode in phase by $5^{\circ}$ and the TRO Y-component signature leads that of the TRO X component by $99^{\circ}$. Since both instruments have a time accuracy of better than 1s, these relative phase measurements have an associated error of $\pm 2^{\circ}$. The TRO Y-component also leads the Doppler O-mode signature by $90^{\circ} \pm 10^{\circ}$, i.e. the TRO X-component is roughly in phase with the Doppler O-mode. The associated error is larger in this case because the phase comparison is made between different data types which have different sample intervals. In addition, the peak-topeak amplitude of Doppler frequency shift normalised to those for the $\mathrm{X}$ - and $\mathrm{Y}$-component ground magnetic signatures $\left(\Delta f / \Delta B_{\mathrm{X}}\right.$ and $\left.\Delta f / \Delta B_{\mathrm{Y}}\right)$ are $0.2 \pm 0.05 \mathrm{~Hz} \mathrm{nT}^{-1}$ $\left(6.7 \pm 1.7 \mathrm{~m} \mathrm{~s}^{-1} \mathrm{nT}^{-1}\right)$ and $0.3 \pm 0.02 \mathrm{~Hz} \mathrm{nT}^{-1}$ $\left(10.8 \pm 0.8 \mathrm{~m} \mathrm{~s}^{-1} \mathrm{nT}^{-1}\right)$, respectively.

In order to determine to what extent the characteristics of the case study presented here are typical of correlated Doppler and magnetometer observations, a quantitative study of a selection of events has been undertaken. In total there were 159 events recorded by the Doppler sounder in the interval from May 1995 (when the sounder commenced measurements) to February 1996, not all of which had ground magnetic signatures. Ten correlated signatures have been selected from this catalogue, chosen because they have the clearest (i.e. least noisy) signatures in the Doppler data and also have both $\mathrm{O}$ - and $\mathrm{X}$-mode signatures. In each case TRO and SOD magnetometer data were utilised in order to determine the $m$-number of each wave.

Parts a and b of Fig. 4 present, respectively, the range of wave frequencies and the onset times of the waves as determined from the Doppler data. Most of the waves had frequencies in the Pc5 range (periods greater than $150 \mathrm{~s}$ ) and they occurred throughout the 03-15 UT interval, peaking around noon. The location of the Doppler sounder means that local time is equal to $\mathrm{UT}+2 \mathrm{~h}$. Figure $4 \mathrm{c}$ indicates the relative phase between the O- and X-mode wave signatures for each event. In all but one of the cases the X-mode signature leads in phase and the average phase shift is $7.5^{\circ}$ with a standard deviation of $8.1^{\circ}$. The $m$-numbers calculated for these events were in the range $3-8$, indicating that they had large azimuthal spatial scale sizes $(1700-4600 \mathrm{~km})$ in the ionosphere above the ground instrumentation.

Figure 5 displays the magnitude of the observed Doppler shifts versus the $\mathrm{X}$ - and $\mathrm{Y}$-component magne-
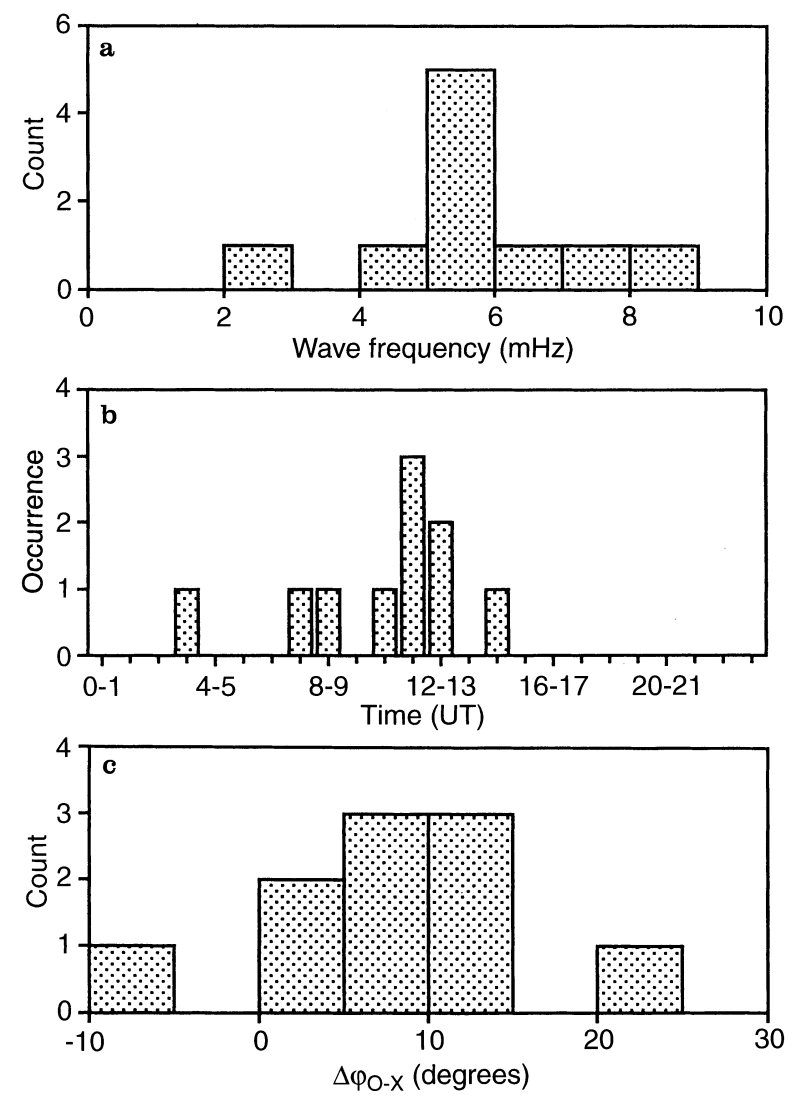

Fig. 4 a-c. Distributions of a ULF wave frequency b diurnal occurrence and $\mathbf{c}$ relative phase between the O- and X-mode Doppler signatures for ten events (see text) where the Doppler signature was accompanied by oscillations in Tromsø magnetometer data. In c, negative relative phase indicates that the O-mode signature was leading in phase

tometer perturbations for each of the correlated events. It indicates that a weak relationship may exist between the magnitudes of the ground and ionospheric pulsa-

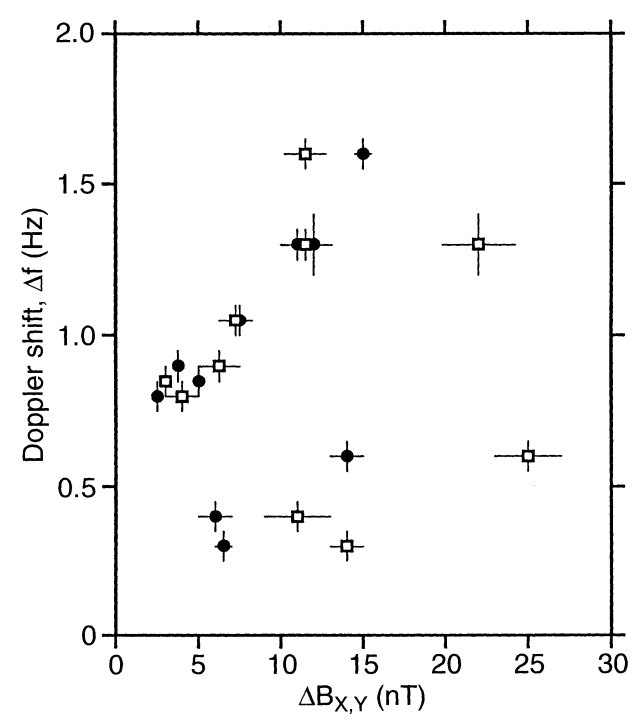

Fig. 5. The O-mode signature peak-to-peak amplitudes as a function of X-component (squares) and Y-component (black circles) peak-topeak deflections observed on the Tromsø magnetometer 


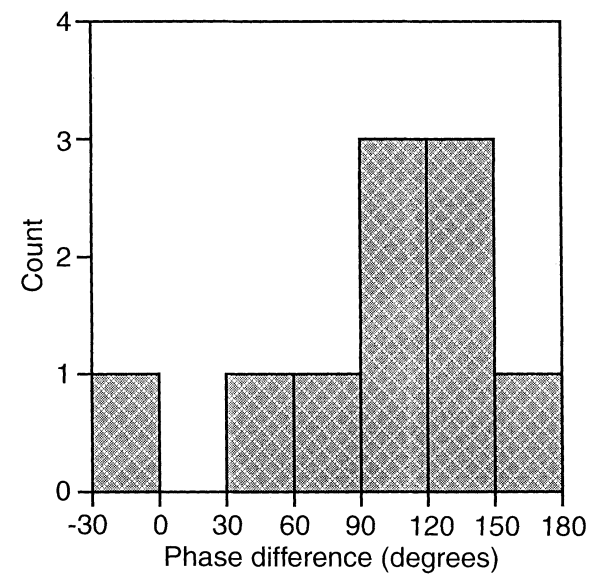

Fig. 6. The occurrence of phase differences between the TRO Ycomponent and the Doppler O-mode ULF wave signatures. Values have been counted in $30^{\circ}$ bins. A positive phase difference indicates a leading TRO Y-component signature

tions, but the data are fairly spread. However, it must be understood that the magnitude of the measured Doppler shifts associated with these waves will be modulated by the changing foF2 throughout the day (see Sect. 2.2 and Fig. 4b). Examination of the magnitude of the peak-topeak Doppler shift (in $\mathrm{Hz}$ ) of an event normalised to the magnitudes (in nT) of the X- and Y-component magnetometer deflections indicates no clear relationship to $m$-number.

The relative phase between Doppler ULF wave signatures and those occurring simultaneously in magnetometer data has been of some interest (e.g. Menk, 1992; Poole et al., 1988; Sutcliffe and Poole, 1990) with regards to the mechanisms which create an ionospheric change associated with the downgoing magnetospheric ULF wave. In this study the relative phase between Doppler O-mode and Y-component magnetometer has been selected. The reason for selecting the $\mathrm{O}$-mode ray is simply that this is the mode which has generally been used by previous authors whether modelling or presenting experimental data. The Y component of the Tromsø magnetometer data has been chosen for this phase comparison, in preference to the $\mathrm{X}$ component for reasons mentioned earlier. The resulting distribution of $B_{Y}$-O-mode phase difference, $\Delta \varphi_{Y O}$, is displayed in Fig. 6. In all but one case the magnetometer data leads the Doppler data in phase, with values of $\Delta \varphi_{\mathrm{YO}}$ typically in the range $90^{\circ}-150^{\circ}$.

\section{Discussion}

\subsection{Source of the observed waves}

At the high-latitude regions of Tromsø, Norway ( $L \approx 6-7)$, ULF waves with a number of distinct driving mechanisms and modes have been observed. Examples of these include field line resonances (e.g. Walker et al., 1979; Yeoman et al., 1990), SABRE (Sweden and Britain Radar-auroral Experiment; Neilsen et al.,
1983) radar observations of equatorwards-propagating Pc5s (Yeoman et al., 1992), and STARE (Scandinavian Twin Auroral Radar Experiment; Greenwald et al., 1978) observations of storm-time Pc5s (Allan et al., 1982, 1983). A number of features of the "correlated" wave signatures presented here suggest that they are the result of magnetohydrodynamic wave modes stimulating field line resonances at or near $L$-shells in the vicinity of Tromsø. The range of observed correlated wave periods is $100-400 \mathrm{~s}(2.5-10 \mathrm{mHz})$ and occur for $3 \leq m \leq 8$, which represents a large azimuthal scale length in the ionosphere. The observed periods are consistent with the findings of Poulter et al. (1984) who presented (see their Fig. 2) the periods associated with field line resonances as a function of geomagnetic latitude derived from STARE VHF radar observations. The range of periods associated with low $m$ field line resonances at the latitude of Troms $\varnothing$ were about 200$590 \mathrm{~s}$ (equivalent to frequencies $1.7-5 \mathrm{mHz}$ ), which is essentially the whole $\mathrm{Pc} 5$ range. In addition, the range of $m$-values for these events agrees with the observations of field line resonances made at slightly lower $L$-shells $(L \approx 4.5-6.6)$ by the SABRE radar and reported by Yeoman et al. (1990).

A characteristic feature of a field line resonance is that the phase of the wave changes by $180^{\circ}$ at either side, in a magnetic meridian, of the resonance region (Southwood, 1974), with the phase poleward of the resonant field line lagging that equatorwards of it (e.g. Orr, 1984). Due to their differing propagation characteristics the $\mathrm{O}$ - and $\mathrm{X}$-mode signals of the Doppler sounder at Tromsø deviate north and south, respectively (e.g. Davies, 1990); Fig. 7 illustrates this effect. Displayed are the O-mode and X-mode ray paths at 4.45 $\mathrm{MHz}$ between the transmitter and receiver locations of the DOPE system (a ground range of $50 \mathrm{~km}$ ). The figure lies in the plane of the magnetic meridian, which in northern Scandinavia is almost coplanar with the geographic meridian, and was generated by the threedimensional ray-tracing program Jones3d (Jones and

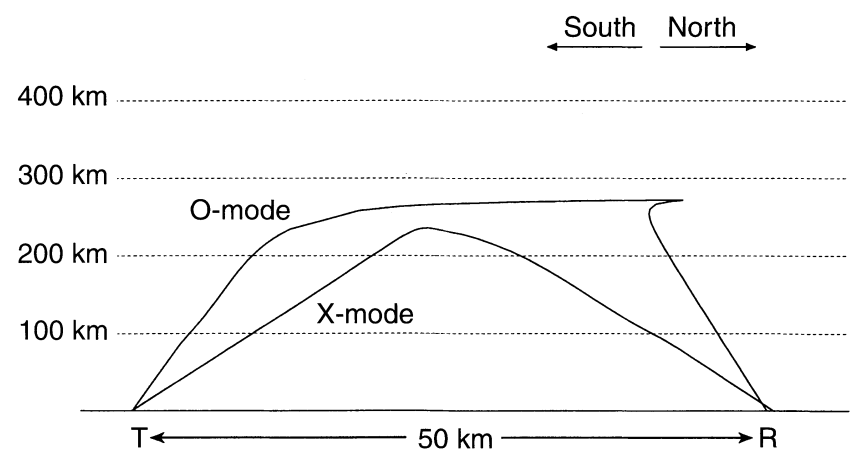

Fig. 7. Output from the three-dimensional ray-tracing program Jones $3 d$ demonstrating the ray paths of the $\mathrm{O}$ - and $\mathrm{X}$-mode radio waves propagated during the DOPE experiment. The figure lies in the plane of the geomagnetic meridian and shows how the O-mode wave deviates towards geomagnetic north and reflects at a higher altitude to that of the $\mathrm{X}$-mode ray. Thus, the wave reflection points are spatially separated. Details of the input parameters to the ray-tracing model are provided in the text 
Stephenson, 1975). The calculation assumed a dipole magnetic field and an ionospheric critical frequency of 4.6 $\mathrm{MHz}$ at $300-\mathrm{km}$ altitude. The O-mode signal is observed to deviate northwards and reflect at a higher altitude compared to the $\mathrm{X}$-mode ray. The Troms $\varnothing$ magnetometer lies close to the magnetic meridian on which the Doppler transmitter and receiver lie and its field of view will encompass the whole region displayed in Fig. 7. Thus, for a ground correlated event, if the signals are field line resonances as suggested here, the Xmode signature phase should lead the O-mode. A typical field line resonance such as that reported by Walker et al. (1979; see their Fig. 14) observed using the STARE radar, had a $180^{\circ}$ phase shift over a latitudinal range of about $1.5^{\circ}$ (equivalent to $1^{\circ} \mathrm{km}^{-1}$ phase change along the ground) in the resonance region. An average phase difference between the $\mathrm{O}$ - and $\mathrm{X}$-mode signals of $7.5^{\circ}$ (Fig. 4c) is, then, equivalent to a horizontal separation of $\sim 7.5 \mathrm{~km}$. This is in reasonable agreement with the results of the ray-tracing presented here, which suggests that an O-X mode separation of $10-20 \mathrm{~km}$ is to be expected. This value is, however, sensitive to the Doppler sounder frequency with respect to the local ionospheric foF 2 and the separation would maximise to a value of about $40-50 \mathrm{~km}$ as the sounder frequency closely approached the critical frequency. The relative phases of the Doppler and magnetometer signatures will be discussed in Sect. 4.3.

\subsection{Previous Doppler observations}

Menk (1992) noted that at low latitudes the Doppler signature of daytime ULF waves was often essentially in phase with the ground magnetic signature but that the relative phase frequently varied throughout the wave packet, which made it difficult to infer any general relationship. He suggested that this was due to phase mixing in the magnetometer signatures from contributions from adjacent regions of the ionosphere. For Pi2 observations in the same data set, no systematic phase relationship could be deduced for waves with periods less than $90 \mathrm{~s}$. However, waves with periods 90-300 s in the Doppler data were generally either directly in or out of phase with ground magnetometer signatures. Menk (1992) demonstrated that the daytime events had values of $\Delta f / \Delta B_{\mathrm{x}}$ in the range $0-0.1 \mathrm{~Hz} \mathrm{nT}^{-1}\left(0-6.5 \mathrm{~m} \mathrm{~s}^{-1} \mathrm{nT}^{-1}\right.$ at $2.3 \mathrm{MHz}$ ) with those for the night-time Pi2s having somewhat smaller values. This compares with the range of $0.02-0.28 \mathrm{~Hz} \mathrm{nT}^{-1}\left(0.7-9.5 \mathrm{~m} \mathrm{~s}^{-1} \mathrm{nT}^{-1}\right.$ at $\left.4.45 \mathrm{MHz}\right)$ which is observed at high latitude for the ten correlated events studied here. The high-latitude results appear to be more phase-stable than those at low latitudes and offer better opportunities for comparison with the modelling work discussed in the next section.

\subsection{Comparison with a numerical model}

Poole et al. (1988) presented work on a model defining the mechanisms which may relate an incident ULF wave to its associated ionospheric Doppler signature. They considerably extended previous models, which considered only a motor effect (Rishbeth and Garriot, 1964; Klostermeyer and Röttger, 1976) where the electric field associated with a geomagnetic pulsation drives an $\boldsymbol{E} \times \boldsymbol{B}$ bulk drift of plasma which, due to the inclination of the field, has a vertical component. This is applicable to Alfvén mode waves with their associated ionospheric magnetic and electric field perturbations. Poole et al. (1988) accounted for changes in refractive index along the radio wave path and also included terms to compensate for magnetic field compression in the ionosphere.

By neglecting source and loss processes for the ionospheric plasma, their model was separated into three main mechanisms. The "magnetic" mechanism accounts for changes in the refractive index due to changes in the magnetic field intensity, requiring no bodily movement of electrons. The "advection" mechanism describes the motor effect on the ionospheric plasma caused by the electric and magnetic perturbations associated with an incident ULF wave. The conversion of the incident Alfvén wave into an evanescent fast mode wave (which is subsequently detected at the ground) in the ionospheric E-region results in compression of the magnetic field, leading to changes in the local plasma density and hence the refractive index. This is the "compression" term, in the nomenclature of Poole et al. (1988) and Sutcliffe and Poole (1989, 1990).

Each mechanism included in the described model contributes a component to the Doppler velocity measured by a sounder. The velocity components may be added vectorially, so that relative phase of the mechanisms is accounted for. It is important to note that the term Doppler velocity here is based on radar convention and in reality may relate more to the effects on the phase path of refractive index changes than actual vertical motions of the reflection point (Poole et al., 1988). So far the model has not been run for conditions at high latitudes, where local source and loss mechanisms for plasma might be important. However, it is possible to make a tentative comparison between high-latitude data presented here and the mid-latitude modelling presented by Sutcliffe and Poole (1990; see their Fig. 3). Although the model predictions given in Sutcliffe and Poole (1990) cover inclination angles and wave frequencies associated with high latitudes, the predictions are based on ionospheric conditions appropriate to a mid-latitude site, and are sensitive to many parameters including season, time of day and magnetic activity.

In the modelling of Sutcliffe and Poole (1989, 1990), the dominant mechanisms for generating the Doppler signature are the advection and compression mechanisms. At higher altitudes (in the F-region), the phase of the component from each mechanism varies slowly with height. This is necessary for the field line resonance interpretation of the north-south phase difference discussed in Sect. 4.1. In the model, velocities and phases are given relative to the magnetic $b_{x}$ and $b_{y}$ components of the ULF wave at the ground which are normalised to amplitudes of $1 \mathrm{nT}$ and $0^{\circ}$ phase. The relative phase 
between the advection and compression effects is height dependent. At F-region heights (above about $150 \mathrm{~km}$ ) they are $180^{\circ}$ out of phase. For a ULF wave with a frequency of $50 \mathrm{mHz}$ (Sutcliffe and Poole, 1990; see their Fig. 1), components of the Doppler velocity due to the advection mechanism lag in phase by about $120^{\circ}$ at Fregion heights and those due to the compressional mechanism lead in phase by about $60^{\circ}$. As the ULF wave frequency falls to $2 \mathrm{mHz}$, these values rise to a lag of $75^{\circ}$ and a lead of $105^{\circ}$, respectively. The data for the correlated events (Fig. 6) indicate that a phase shift. $\Delta \varphi_{\mathrm{YO}}$, in the range $90^{\circ}-150^{\circ}$ occurred most often, with $b_{y}$ leading in phase. Thus, the model, which is derived for an ionospherically reflected downgoing Alfvén wave, implies that the advection mechanism dominates during these events and that field compression is small, a fact which is supported by the observations reported here. In addition to the work presented in this paper and that by Menk (1992), there have been other reports of measurements of the phase difference between the ionospheric Doppler signature and the ground magnetic wave, but these are fairly few and inconclusive. Sutcliffe and Poole (1984) showed that the ionospheric pulsation signature of two events observed at low latitude with a chirp sounder had been the result of equal contributions from both changes in the phase path associated with variations in the refractive index caused by magnetic field oscillations and an advective component. The digisonde measurements at Halley reported by Jarvis and Gough (1988) again indicated that the phase relationship was a complicated one and that compressional and advective processes were both important. Similarly, the nine events recorded by low-latitude spaced HF Doppler sounders and presented by Tedd et al. (1989) showed a highly variable nature. They came to the same conclusions as Jarvis and Gough (1988). Measurements of the phases of ionospheric signatures of Pc3s made using the EISCAT UHF radar reported by Glangeaud et al. (1985) required intensive signal analysis to improve the system signal to noise ratio and then the frequency (and thus phase) was found to very variable over short timeintervals.

The amplitude of the Doppler oscillations relative to the peak-to-peak magnitude of the magnetometer signature in the north-south $(\mathrm{X})$ component for the correlated events in this study are in the range $0.02-0.28$ $\mathrm{Hz} \mathrm{nT}^{-1}\left(0.7-9.5 \mathrm{~m} \mathrm{~s}^{-1} \mathrm{nT}^{-1}\right.$ at $\left.4.45 \mathrm{MHz}\right)$. These values are considerably larger than those predicted by the model which, at F-region heights are expected to be less than $1 \mathrm{~m} \mathrm{~s}^{-1} \mathrm{nT}^{-1}$. The likely reason for the discrepancy is twofold. Firstly, the north-south scale length of the incident ULF wave employed in the model was $500 \mathrm{~km}$ which, as noted by Menk (1992), is likely to be too large. Field line resonances are known to be highly localised in latitude [e.g. the recent study of Yeoman et al. (1997) suggested a scale length of only $60 \mathrm{~km}$ ] and a scale length of $80-100 \mathrm{~km}$ would be a more accurate input to the model. This would increase the amount of phase mixing (from adjacent regions of the ionosphere) imposed on the magnetometer signature and would correspondingly increase the relative amplitude of the
Doppler velocity predicted by the model. Secondly, the effective amplification of signatures observed by the Doppler sounder at a frequency close to the foF2 will also have a significant bearing on the comparison. The effect may complicate a comparison of observed and modelled values.

\section{Summary}

A number of ionospheric signatures of magnetospheric ULF waves have been successfully observed with a new HF Doppler sounder, DOPE, recently deployed near Tromsø, Norway. Clear correlation of these signatures has been achieved with ULF waves recorded in ground magnetometer data and a quantitative analysis has been performed on the ten clearest events in the Doppler data.

The "correlated" events exhibit wave periods in the range 100-400 $\mathrm{s}$ and azimuthal wave numbers in the range $3 \leq m \leq 8$. This is consistent with previous observations on the ground, in the ionosphere and in the magnetosphere of the signatures associated with field line resonances with low $m$-values (large azimuthal scale sizes). In addition, the relative phases between the Oand X-mode Doppler signatures is indicative of the latitudinal phase profile of a field line resonance. These phase measurements contribute towards the verification of a model of the conversion mechanisms between the magnetospheric pulsation electric and magnetic field perturbations of an incident Alfvén wave and its ionospheric signature in a Doppler sounder (Poole et al., 1988; Sutcliffe and Poole, 1989, 1990; Sutcliffe, 1994). The results imply that the "advection" mechanism, as defined in the foregoing model, is dominant in the generation of the ionospheric Doppler signatures of high-latitude ULF waves, in the Pc4-5 frequency range, with large spatial scale sizes.

There is need to collect sufficient data to verify more thoroughly the model of Poole et al. (1988), Sutcliffe and Poole (1989, 1990) and Sutcliffe, (1994). Application of the model should enable the determination of which mechanisms contribute the most to the ionospheric changes observed by the Doppler sounder, and under what conditions these contributions occur. The model will need to be run with input parameters and profiles closer to those appropriate for the present level of solar activity and for high-latitude conditions. The results presented here are the first data from the DOPE experiment. However, the sounder is in continuous operation in the Norwegian Arctic and is expected to collect data over a period of several years. There already exists a limited data base of EISCAT measurements from the SP-UK-DOPE program. Such data will provide measurements of ion flows and E-region conductivities in the ionosphere. These can be input to the model to investigate the evolution of an incident ULF wave in the ionosphere and the boundary conditions associated with it. The evolution of the ULF wave signatures and the reflection and damping of ULF waves have been the subject of some previous modelling 
studies. Unfortunately, these have been poorly tested experimentally due to the difficulty in making appropriate measurements. It is anticipated that HF Doppler sounders will play an important role in these future observations.

Acknowledgements. The authors wish to thank the Royal Society for funding the DOPE project and the Particle Physics and Astronomy Research Council for support during the work presented in this paper; Stuart Crooks and Michael Parsons, technical staff of the Radio and Space Plasma Physics group who were involved in the construction of the sounder; Michael Reitveld and Stein Eliassen at the EISCAT site in Ramfjordmoen for looking after the DOPE sounder and for returning data tapes to the UK; the Sodankylä Geophysical Observatory and Børre Holmeslet at the Tromsø Auroral Observatory for providing magnetometer data.

Topical Editor K.-H. Glaßmeier thanks F. Menk and M. J. Jarvis for their help in evaluating this paper.

\section{References}

Allan, W., E. M. Poulter, and E. Nielsen, STARE observations of a Pc5 pulsation with large azimuthal wave number, J. Geophys. Res., 87, 6163, 1982.

Allan, W., E. M. Poulter, and E. Nielsen, Pc5 pulsations associated with ring current proton drifts: STARE radar observations, Planet. Space Sci., 31, 1279, 1983.

Chapman, P. J., A cost effective frequency synthesizer for Doppler applications, Radio and Space Plasma Physics Group Tech. Rep. 60, Leicester University, UK, 1995.

Chapman, P. J., HF Doppler receiver, Radio and Space Plasma Physics Group Tech. Rep. 63, Leicester University, UK, 1997a.

Chapman, P. J., HF Doppler transmitter, Radio and Space Plasma Physics Group Tech. Rep. 64, Leicester University, UK, $1997 \mathrm{~b}$.

Crowley, G., N. Wade, J. A. Waldock, T. R. Robinson, and T. B. Jones, High time-resolution observations of periodic frictional heating associated with a Pc5 micropulsation, Nature, 316, 528, 1985.

Crowley, G., W. J. Hughes, and T. B. Jones, Observational evidence of cavity modes in the Earth's magnetosphere, J. Geophys. Res., 92, 12233, 1987.

Davies, K., Ionospheric radio, IEE Electromagnetic Waves series 31, Peter Peregrinus, London, 1990.

Fenwick, R. C., and O. G. Villard, Continuous recordings of frequency variation of the WWV-20 signal after propagation over a 4000-km path, J. Geophys. Res., 67, 3249, 1960.

Georges, T. M., Ionospheric effects of atmospheric waves, E.S.S.A. Tech. Rep., Inst. Environ. Res. (Boulder, Colo.), I.E.R. 57I.T.S.A. 54, October 1967.

Glangeaud, F., C. Lathuillere, M. Lambert, and Z.Y. Zhao, Pc 3-4 ULF magnetic variations measured in the ionosphere by EISCAT, J. Geophys. Res., 90, 8319, 1985.

Greenwald, R. A., W. Weiss, E. Nielson, and N. P. Thomson, STARE: a new radar auroral backscatter experiment in northern Scandinavia, Radio Sci., 13, 1021, 1978.

Hughes, W. J., Hydromagnetic waves in the magnetosphere, in Solar Terrestrial Physics, Eds. R. L. Carovillano, and J. M. Forbes), Reidel, Dordrecht, 1983.

Hughes, W. J., and D. J. Southwood, The screening of micropulsation signals by the atmosphere and ionosphere, J. Geophys. Res., 81, 3234, 1976.

Jacobs, J. A., and T. Watanabe, Doppler frequency changes in radio waves propagating through a moving ionosphere, Radio Sci., 1, 257, 1966.

Jacobs, J. A., Y. Kato, S. Matsushita, and V. A. Troitskaya, Classification of geomagnetic micropulsations, J. Geophys. Res., 69, 180, 1964
Jarvis, M. J., and H. Gough Digital ionosonde observations of Pc3-4 pulsations across the plasmapause, Planet. Space Sci., 36, 733, 1988

Jones, R. M., and J. J. Stephenson, A versatile 3D raytracing computer program for radio waves in the ionosphere, OT-Rep75-76, US. Gov. Print., Washington DC, 1975.

Klostermeyer, J., and J. Röttger, Simultaneous geomagnetic and ionospheric oscillations caused by hydromagnetic waves, Planet. Space Sci., 24, 1065, 1976.

Lühr, H., The IMAGE magnetometer network, STEP Int. Newslett. 4, 4, 1994.

Menk, F. W., Characterization of ionospheric Doppler oscillations in the PC3-4 and Pi2 pulsation frequency range, Planet. Space Sci., 40, 495, 1992.

Nielsen, E., W. Guttler, E. C. Thomas, C. P. Stewart, T. B. Jones, and A. Hedburg, SABRE: a new radar auroral backscatter experiment, Nature, 304, 712, 1983.

Orr, D., Magnetospheric hydrodynamic waves: their eigenperiods, amplitudes and phase variations: a tutorial introduction, J. Geophys., 55, 76, 1984.

Poole, A. W. V., P. R. Sutcliffe, and A. D. M. Walker, The relationship between ULF geomagnetic pulsations and ionospheric Doppler oscillations: derivation of a model, J. Geophys. Res., 93, 14656, 1988.

Poulter, E. M., W. Allan, J. G. Keys, and E. Nielsen, Plasmatrough ion mass densities determined from ULF pulsation eigen periods, Planet. Space Sci., 32, 1069, 1984.

Rishbeth, H., and O. K. Garriot, Introduction to ionospheric physics, Academic Press, New York, London, 1964.

Rishbeth, H., and P. J. S. Williams, The EISCAT ionospheric radar: the system and its early results, $Q$. J. R. Astron. Soc., 26, 478, 1985.

Ruohoniemi, J. M., R. A. Greenwald, K. B. Baker, and J. C. Samson, HF radar observations of Pc5 field line resonances in the midnight/early morning MLT sector, J. Geophys. Res., 96, 15697, 1991.

Southwood, D. J., Some features of field line resonances in the magnetosphere, Planet. Space Sci., 22, 483, 1974.

Sutcliffe, P. R., Modelling the ionospheric signatures of geomagnetic pulsations, J. Geomagn. Geoelectr., 46, 1011, 1994.

Sutcliffe, P. R., and A. W. V. Poole, Low-latitude Pc3 pulsations and associated ionospheric oscillations measured by a digital chirp ionosonde, Geophys. Res. Lett., 11, 1172, 1984.

Sutcliffe, P. R., and A. W. V. Poole, Ionospheric Doppler and electron velocities in the presence of ULF waves, J. Geophys. Res., 94, 13505, 1989.

Sutcliffe, P. R., and A. W. V. Poole, The relationship between ULF geomagnetic pulsations and ionospheric Doppler oscillations: model predictions, Planet. Space Sci., 38, 1581, 1990.

Tedd, B. L., K. D. Cole and P. L. Dyson, The association between ionospheric and geomagnetic pulsations in the Pc3-4 range at mid-latitudes, Planet. Space Sci., 37, 1079, 1989.

Walker, A. D. M., R. A. Greenwald, W. F. Stuart, and C. A. Green, STARE auroral radar observations of Pc5 geomagnetic pulsations, J. Geophys. Res., 84, 3373, 1979.

Wright, D. M., HF Doppler observations of ULF waves: system development and high-latitude results, Ph.D. thesis, Univ. Leicester, UK, August 1996.

Yeoman, T. K., M. Lester, D. Orr, and H. Lühr, Ionospheric boundary conditions of hydromagnetic waves: the dependence on azimuthal wave number and a case-study, Planet. Space Sci., 38, 1315, 1990.

Yeoman, T. K., Mao Tian, M. Lester, and T. B. Jones, A study of Pc5 hydromagnetic waves with equatorward phase propagation, Planet, Space Sci., 40, 797, 1992.

Yeoman, T. K., D. M. Wright, T. R. Robinson, J. A. Davies, and M. T. Reitveld, High spatial and temporal resolution observations of an impulse-driven field line resonance in radar backscatter artificially generated with the Tromsø heater, Ann. Geophysicae, 15, 634, 1997. 